\title{
Tips for Avoiding Artifacts from UV Photoconversion of DAPI
}

\section{Lori M. Roberts}

Biotium Inc., 46117 Landing Parkway, Fremont, CA 94538

lroberts@biotium.com

\begin{abstract}
Exposure of the nuclear counterstain DAPI to just a few seconds of UV excitation light during fluorescence imaging can photoconvert the dye to stable green- and red-emitting forms, resulting in imaging artifacts in multi-color staining experiments. This article reviews the published literature on photoconversion and compares the effect of different mounting media on photoconversion of DAPI in stained tissue sections. Once photoconversion is recognized as a potential source of non-specific fluorescence, careful imaging practices or reagent selection can be used to avoid it.
\end{abstract}

Keywords: DAPI, Hoechst, photoconversion, bleed-through, cross talk

\section{Introduction}

Fluorescence imaging offers a major advantage over brightfield microscopy because it allows multiplex detection of several targets in the same specimen. Using spectrally well-separated dyes, multiple targets within the same sample can be stained or labeled. Ultraviolet (UV) or laser light is then used to excite these dyes to fluorescence, permitting target identification by color using multicolor fluorescence imaging. Since their introduction in the 1970s, diamidino-2-phenylindole (DAPI) and Hoechst dyes have been the nuclear counterstains of choice for immunofluorescence staining. Bright, nuclear-selective, and easy-to-use, the dyes occupy the blue fluorescence channel that is frequently sub-optimal for detecting other targets because of the high intrinsic blue background fluorescence of most cells and tissues. However, DAPI and Hoechst dyes can present some pitfalls for fluorescence imaging. In particular, their signal can bleed through into the green channel because of their broad fluorescence emission, leading to imaging artifacts. More recently, another source of fluorescence cross talk of DAPI and Hoechst dyes has been described [1]; this is the photoconversion of the dyes by UV light to generate fluorophores with green and red emission. This spurious emission could interfere with specific imaging of green and red fluorescent targets, especially because focusing on blue stained nuclei in the specimen is often the microscopist's first step during imaging.

The photoconversion of DAPI by UV exposure to generate green fluorescence was described by Piterburg and colleagues in 2012 [1]. Other groups have expanded upon this observation, showing that both DAPI and Hoechst dyes undergo photoconversion and that different fixation methods and mounting media can enhance the process [2]. Both green and red fluorescent products have been detected after UV exposure of DAPI and Hoechst [3]. Photoconversion of Hoechst also has been shown to occur with $405 \mathrm{~nm}$ laser excitation, a potential complication for studies using Hoechst dyes to photosensitize living cells to UV-induced DNA damage [4].

Photoconversion has been reported for DAPI, Hoechst dyes, and Vybrant ${ }^{\circledR}$ DyeCycle $^{\mathrm{TM}}$ Violet in the presence or absence of DNA [5]. Multiple groups reported that the appearance of photoconversion products correlated with the photobleaching of blue fluorescence and that while blue fluorescence recovered over time in the dark, the photoconversion products did not disappear completely $[3,5,6]$. Żurek-Biesiada and colleagues examined the chemical mechanisms of photoconversion. Exposure to hydrogen peroxide mimicked the effect of UV light, suggesting that photoconversion was the result of photooxidation. Mass spectrometry analysis revealed that the green fluorescent species that was generated, by peroxide or UV treatment of DAPI or Hoechst, was a protonated form of the dye; however, the $\mathrm{pH}$ of the environment did not influence photoconversion [5]. A summary of the literature describing photoconversion of DAPI and similar dyes is provided in Table 1.

Once researchers are aware of potential artifacts from photoconversion of DAPI and similar dyes, careful imaging practices can be used to minimize UV excitation of dyes before image capture. In addition, it is essential to design appropriate negative control experiments for detecting and avoiding fluorescence cross talk from a variety of sources.

\section{Materials and Methods}

Tissue staining. Rat skeletal muscle cryosections were purchased from Zyagen (San Diego, CA). DAPI, mounting media, and coverslip sealant were from Biotium (Fremont, CA). Cryosections were fixed in ice-cold methanol for 5-10 minutes at $-20^{\circ} \mathrm{C}$ and then rinsed three times with phosphate-buffered saline (PBS). Sections were stained with $1.5 \mu \mathrm{g} / \mathrm{mL}$ DAPI in PBS for 3 minutes at room temperature and then rinsed once with PBS. Sections were mounted using PBS (with no antifade compounds added), EverBrite Mounting Medium, Dropn-Stain EverBrite Mounting Medium, or EverBrite Hardset Mounting Medium and placed under coverslips. The coverslip edges were sealed with CoverGrip Coverslip Sealant. EverBrite Hardset was allowed to cure overnight at room temperature, protected from light. Slides were stored at $4^{\circ} \mathrm{C}$ before imaging.

Fluorescence imaging. Specimens were imaged using a Zeiss LSM700 laser scanning confocal imaging system attached to an Axio Observer Z1 inverted fluorescence microscope (Carl Zeiss, Oberkochen, Germany) with an X-Cite 120-Watt short arc lamp (EXTO Life Sciences, Mississauga, Ontario, Canada). Light exposure and imaging were performed through a Zeiss ECPlan-NEOFLUAR 40×/1.3 DIC oil objective. For UV exposure, specimens were exposed to UV excitation through a $365 \mathrm{~nm}$ DAPI filter (Zeiss filter set 49), with the lamp controller set to the highest brightness setting. For $405 \mathrm{~nm}$ laser exposure, specimens were excited using the confocal system's $5 \mathrm{~mW} 405 \mathrm{~nm}$ solid-state laser at $10 \%$ power or $90 \%$ power setting. Images 


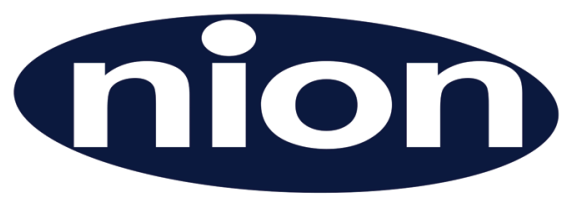

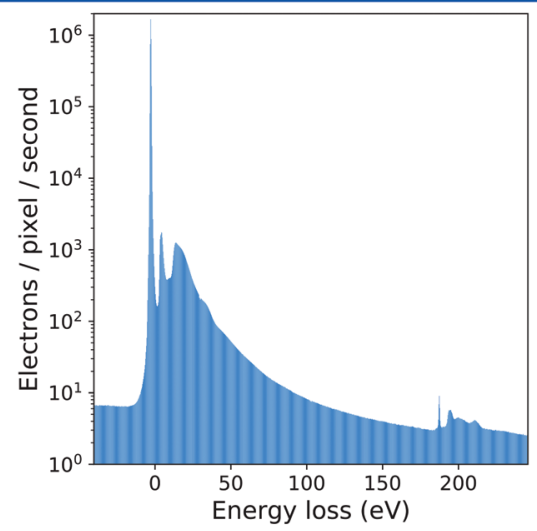

EELS of hexagonal boron nitride (h-BN). $E_{0}=100 \mathrm{keV}$, beam current=170 pA, 10s exposure, $0.28 \mathrm{eV} / \mathrm{ch}$. Raw data (no dark subtraction or gain normalization).

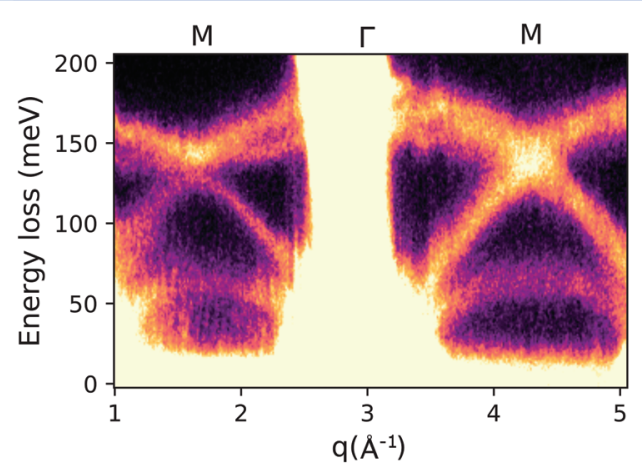

Momentum-resolved EELS of h-BN showing phonon dispersion curves $S(q, \omega)$ acquired in 6.7 minutes (100 frames $\times 4$ seconds, aligned) using a slot entrance aperture. $E_{0}=60 \mathrm{keV}$, beam current=10 pA, energy resolution $10 \mathrm{meV}$.

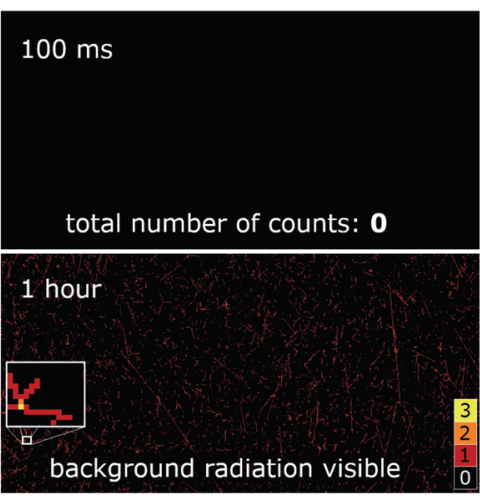

Detector sensitivity test with no signal. Top: With short exposure times, there is no background. Bottom: With long exposure times, cosmic rays (typically muons) are detected.

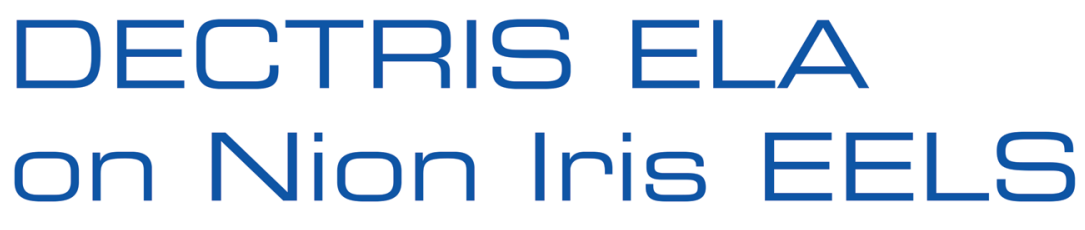

A new direct detector
for $30-200 \mathrm{keV}$ EELS

\section{DECTRIS hybrid pixel detector}

- $1030 \times 516$ pixels of $75 \mu \mathrm{m}$ each

- 2,250 full frames/s and faster for sub-areas

- PSF @ 100 keV 1.3 pixels, better at lower keV

- DQE 0.8 (without super-resolution)

- Dynamic range $>10^{6}: 1$

- Can use >100 pA while counting single electrons

- Optimized for 30-200 keV operation 
Table 1: Comparison of published reports of photoconversion of DAPI and similar nuclear stains. First column shows references.

\begin{tabular}{|c|c|c|c|c|c|c|c|c|}
\hline Ref. & $\begin{array}{l}\text { Imaging system/ } \\
\text { irradiation } \\
\text { source }\end{array}$ & Specimen & $\begin{array}{l}\text { UV exp. } \\
\text { time }\end{array}$ & Dyes tested & $\begin{array}{l}\text { Dye } \\
\text { concen- } \\
\text { tration }\end{array}$ & $\begin{array}{l}\text { Fixation } \\
\text { method }\end{array}$ & $\begin{array}{l}\text { Mounting } \\
\text { media }\end{array}$ & $\begin{array}{l}\text { Notes on } \\
\text { photoconversion }\end{array}$ \\
\hline 1 & $\begin{array}{l}\text { Nikon TE2000E, } \\
\text { 40×/NA } 1.3 \text { oil } \\
\text { objective, } \\
350 / 50 \mathrm{~nm} \text { or } \\
400 / 30 \mathrm{~nm} \\
\text { excitation filter }\end{array}$ & $\begin{array}{l}\text { B16 cells on } \\
\text { coverglass }\end{array}$ & $\geq 2 \min$ & DAPI & $\begin{array}{l}0.14 \text { or } \\
1.4 \mu \mathrm{m} \\
\text { DAPI }\end{array}$ & $\begin{array}{l}\mathrm{MeOH} \text { or } \\
\text { PFA }\end{array}$ & $\begin{array}{l}\text { Eukitt } \AA \text {, } \\
\text { Fluoro- } \\
\text { mount-G®, } \\
\text { or } \\
\text { Prolong } \AA \\
\text { Gold }\end{array}$ & $\begin{array}{l}\text { - Occurred with } 350 \text { and } \\
400 \text { nm filters } \\
\text { - Independent of dye } \\
\text { concentration } \\
\text { - Occurred with oil or air } \\
\text { objective } \\
\text { - Higher with } \mathrm{MeOH} \\
\text { fixation or Eukitt@ } \\
\text { mountant }\end{array}$ \\
\hline 2 & $\begin{array}{l}\text { Zeiss Axio } \\
\text { Observer.Z1, } \\
63 \times \text { air objective, } \\
100 \mathrm{~W} \text { Hg arc } \\
\text { lamp, } 365 \mathrm{~nm} \\
\text { excitation filter }\end{array}$ & $\begin{array}{l}\text { NTERA-2 cells } \\
\text { on coverglass }\end{array}$ & $\begin{array}{l}1-64 \\
\text { sec. }\end{array}$ & $\begin{array}{l}\text { DAPI } \\
\text { Hoechst } \\
33258 \\
\text { Hoechst } \\
33342\end{array}$ & $\begin{array}{l}0.1-1 \mu \mathrm{m} \\
\text { DAPI } \\
1 \mu \mathrm{m} \\
\text { Hoechst }\end{array}$ & $\begin{array}{l}\text { Accustain } \AA \text {, } \\
\text { MeOH, or } \\
\text { PFA }\end{array}$ & $\begin{array}{l}\text { Glycerol } \\
(50 \%, 80 \% \text {, } \\
\text { or } 100 \%) \text { or } \\
\text { PBS }\end{array}$ & $\begin{array}{l}\text { - Correlated with higher } \\
\text { glycerol concentration } \\
\text { - Higher with Accustain }{ }^{\circledR} \\
\text { fixative } \\
\text { - Lowest with Hoechst } \\
33342 \text { with } \leq 80 \% \\
\text { glycerol } \\
\text { - No appearance of green } \\
\text { fluorescence in } \\
\text { unstained cells after UV } \\
\text { exposure }\end{array}$ \\
\hline 3 & $\begin{array}{l}\text { Olympus DSU, } \\
60 \times / N A 1.42 \text { oil } \\
\text { objective, } 100 \mathrm{~W} \\
\text { Hg arc lamp, UV/ } \\
\text { blue filter cube }\end{array}$ & $\begin{array}{l}\text { Drosophila } \\
\text { testis,metaphase } \\
\text { chromosome } \\
\text { squashes }\end{array}$ & 3-7 min. & $\begin{array}{l}\text { DAPI } \\
\text { Hoechst } \\
33258 \\
\text { Hoechst } \\
33342\end{array}$ & $\begin{array}{l}7.2 \mu \mathrm{m} \\
\text { DAPI } \\
81 \mu \mathrm{m} \\
\text { Hoechst }\end{array}$ & $\begin{array}{l}\text { PFA (testis) } \\
\mathrm{MeOH} / \\
\mathrm{AcOH} \\
\text { (chromo- } \\
\text { somes) }\end{array}$ & $\begin{array}{l}\text { VECTA- } \\
\text { SHIELD® } \\
\text { or } \\
\text { SlowFade } \AA \\
\text { Gold }\end{array}$ & $\begin{array}{l}\text { - Green and red fluores- } \\
\text { cence appeared at the } \\
\text { same time that blue fluo- } \\
\text { rescence bleached } \\
\text { - Equivalent for all dyes } \\
\text { tested, even though } \\
\text { Hoechst } 33258 \\
\text { bleached more rapidly } \\
\text { than DAPI } \\
\text { - Blue fluorescence } \\
\text { recovered over time in } \\
\text { dark, but green/red } \\
\text { fluorescence remained }\end{array}$ \\
\hline 4 & $\begin{array}{l}\text { Olympus IX81, } \\
\text { 100×/NA } 1.45 \\
\text { TIRF oil objective, } \\
\text { Visitron VisiFRAP } \\
405 \mathrm{~nm} \text { laser } \\
\text { (12.8 mW at } \\
\text { objective) }\end{array}$ & Live U2OS cells & $\begin{array}{l}\text { Not } \\
\text { speci- } \\
\text { fied }\end{array}$ & $\begin{array}{l}\text { Hoechst } \\
33342\end{array}$ & $\begin{array}{l}2.4 \mu \mathrm{m} \\
\text { DAPI }\end{array}$ & None & $\mathrm{N} / \mathrm{A}$ & $\begin{array}{l}\text { - A particular concern } \\
\text { when Hoechst used as a } \\
\text { photosensitizer for } \\
\text { UV-induced damage }\end{array}$ \\
\hline 5 & $\begin{array}{l}\text { Leica EL6000, } \\
63 \times / N A 1.4 \text { oil } \\
\text { objective, Hg } \\
\text { metal halide lamp } \\
(11 \mathrm{~mW}) \\
360 / 40 \mathrm{~nm} \\
\text { excitation filter }\end{array}$ & $\begin{array}{l}\text { MSU } 1.1 \text { cells on } \\
\text { coverglass }\end{array}$ & $1 \mathrm{~min}$. & $\begin{array}{l}\text { Hoechst } \\
33258 \\
\text { DAPI } \\
\text { Vybrant }{ }^{\circledR} \\
\text { DyeCycle } \\
\text { Violet }\end{array}$ & $\begin{array}{l}1 \mu \mathrm{m} \text { DAPI } \\
3.2 \mu \mathrm{m} \\
\text { Hoechst } \\
1 \mu \mathrm{m} \\
\text { DyeCy- } \\
\text { cle }^{\mathrm{TM}}\end{array}$ & $\begin{array}{l}\text { Fixed } 4 \% \\
\text { PFA, stored } \\
\text { in 1\% PFA } \\
\text { before } \\
\text { staining }\end{array}$ & None & $\begin{array}{l}\text { - Higher with Hoechst } \\
\text { than DAPI } \\
\text { - Green and red fluores- } \\
\text { cence appeared at the } \\
\text { same time that blue fluo- } \\
\text { rescence bleached } \\
\text { - Independent of DNA, } \\
\text { dye concentration, or pH } \\
\text { - Blue fluorescence } \\
\text { recovered over time in } \\
\text { dark, but green/red } \\
\text { fluorescence remained } \\
\text { - No appearance of green } \\
\text { fluorescence in } \\
\text { unstained cells after UV } \\
\text { exposure } \\
\text { - } \mathrm{H}_{2} \mathrm{O}_{2} \text { mimicked effect of } \\
\text { UV, and both caused } \\
\text { dye protonation }\end{array}$ \\
\hline 6 & $\begin{array}{l}\text { Zeiss Axio- } \\
\text { Vert200M, } 63 \times / \\
\text { NA } 1.4 \text { oil } \\
\text { objective, } 100 \mathrm{~W} \\
\text { Hg bulb }\end{array}$ & Fixed $\mathrm{CHO}$ cells & $\begin{array}{l}1-200 \\
\text { sec. }\end{array}$ & DAPI & $\begin{array}{l}3.6 \mu \mathrm{m} \\
\text { DAPI }\end{array}$ & $\begin{array}{l}\text { Not } \\
\text { specified }\end{array}$ & $\begin{array}{l}\text { Prolong }{ }^{\circledR} \\
\text { Gold }\end{array}$ & $\begin{array}{l}\text { - Correlated with UV } \\
\text { intensity and exposure } \\
\text { time }\end{array}$ \\
\hline
\end{tabular}


were scanned with laser power at $10 \%$. The DAPI channel was scanned first, and then the fluorescein isothiocyanate (FITC) and tetramethylrhodamine isothiocyanate (TRITC) channels were scanned. After exposure of a field to UV or $405 \mathrm{~nm}$ light, the stage was moved to image a new field of view with low green or red autofluorescence, using $488 \mathrm{~nm}$ and $555 \mathrm{~nm}$ excitation for subsequent imaging. Images shown in Figure 3 were captured at $1 \times$ zoom; all other images were captured at $0.5 \times$ zoom. Three different fields were captured for each experimental condition. Laser power and gain settings were kept constant for comparison of fluorescence intensity between images.

\section{Results}

Photoconversion rates. In agreement with published studies, UV excitation of DAPI-stained skeletal muscle tissue sections in wet-set mounting medium resulted in rapid appearance of green and red fluorescent photoconversion products. Neither green nor red nuclear fluorescence was detected in areas of the tissue that had not been exposed to UV light through the DAPI filter cube (Figures 1E and 1I). However, after less than 5 seconds of UV exposure, green and red nuclear fluorescence could be observed (not shown). The green and red nuclear fluorescence became readily detectable after 10 seconds of UV exposure (Figures $1 \mathrm{~F}$ and $1 \mathrm{~J}$ ) and increased in intensity with longer UV exposure times of up to 60 seconds (Figures $1 \mathrm{H}$ and 1L). The fluorescence intensity in the green and red channels was roughly equal. When the specimen was moved to an adjacent field of view, no green/red nuclear fluorescence was observed in areas of the section that were outside the UV-exposed field (not shown).

Photoconversion products. Merged images of DAPI (blue) and TRITC (red) channels are shown in Figure 2 to

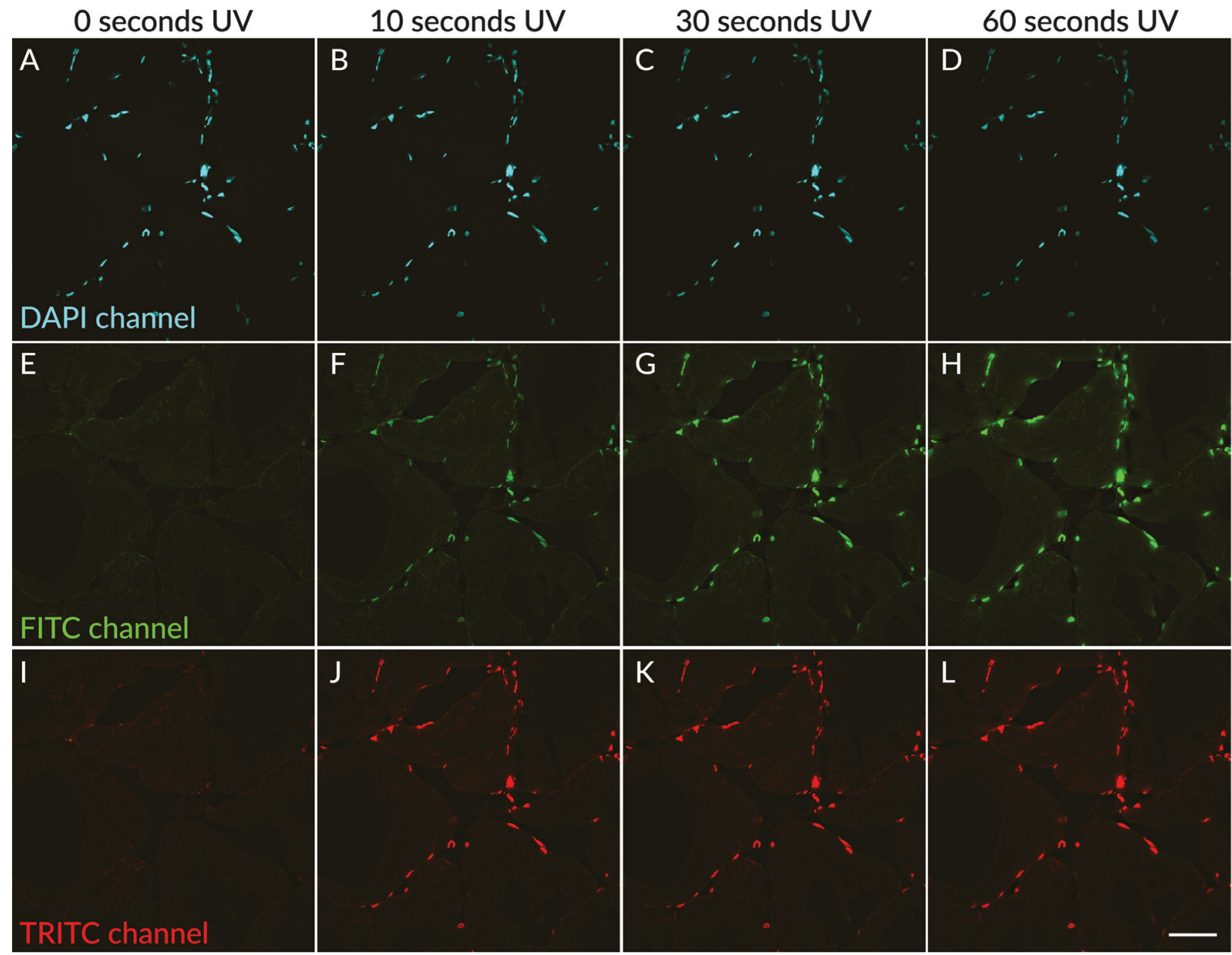

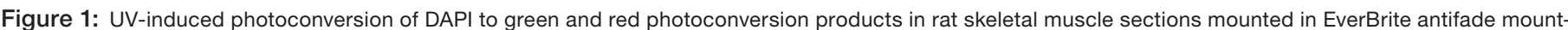

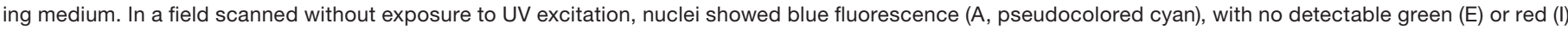

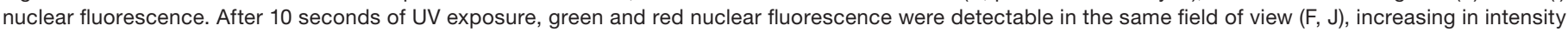
after 30 seconds $(G, K)$ and 60 seconds $(H, L)$ of UV exposure, with concurrent dimming of nuclear fluorescence in the DAPI channel $(B-D)$. Scale bar $=50 \mu m$. 
No UV

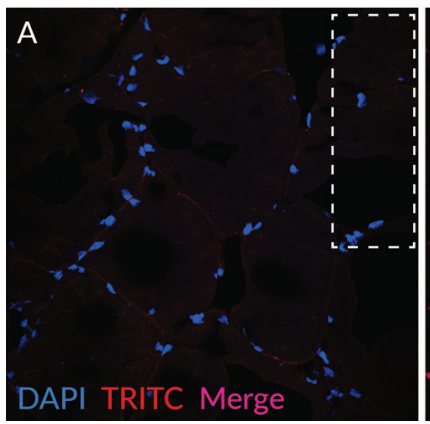

30 seconds UV

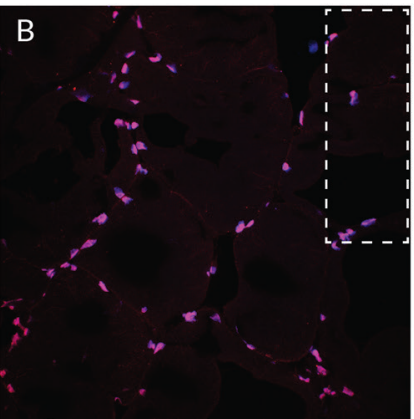

Adjacent field

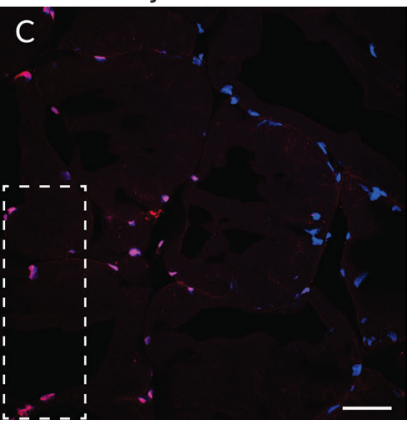

Figure 2: Photoconversion of DAPI was detectable only in areas exposed to UV excitation. (A) Merged image of DAPI (blue) and TRITC (red) channels in a field scanned without UV excitation, showing blue nuclear fluorescence without detectable red fluorescence. (B) The same field as in (A), after 30 seconds of UV exposure, showing red photoconversion product, which appears pink to red when merged with the blue DAPI signal depending on the intensity of the red fluorescence. (C) A field adjacent to that in (A) and (B), showing nuclei adjacent to the area exposed to UV. Nuclei with only blue detectable fluorescence can be imaged alongside nuclei with both blue DAPI fluorescence and red photoconverted fluorescence. The dashed outlines indicate the position of the same region of the tissue in the different fields of view. Scale bar $=50 \mu \mathrm{m}$.

fluorescence (not shown). The photoconversion products appeared to be stable because areas with green and red fluorescent nuclear fluorescence were readily detectable in the specimen when the slide was stored at $4^{\circ} \mathrm{C}$ in the dark for several days after initial UV exposure (not shown).

Laser excitation. In contrast to UV excitation, exposure of sections to $405 \mathrm{~nm}$ laser excitation did not result in rapid appearance of photoconversion products. After $60 \mathrm{sec}-$ onds of excitation at $10 \%$ laser power (the intensity used for image capture in these experiments), photoconversion products were not detectable (Figure 3F). Subsequent excitation show the color differences of nuclear staining before (Figure 2A) and after (Figure 2B) photoconversion. Nuclei with red photoconversion product appear pink to red in the merged images depending on the intensity of the photoconversion product, while nuclei in the field adjacent to the UV-exposed area without photoconversion product appear blue (Figure 2C). Green fluorescence of the photoconversion product was also observed and had similar intensity as the red of the same field of view with $405 \mathrm{~nm}$ laser at $90 \%$ power for 60 seconds resulted in the appearance of dim photoconversion product fluorescence (Figure 3G) that was much less intense than the fluorescence that resulted from exposure of the same field of view to UV excitation for 30 seconds (Figure $3 \mathrm{H}$ ). Red nuclear fluorescence also was observed after UV and $405 \mathrm{~nm}$ exposure, which had similar intensity as the green fluorescence (not shown).

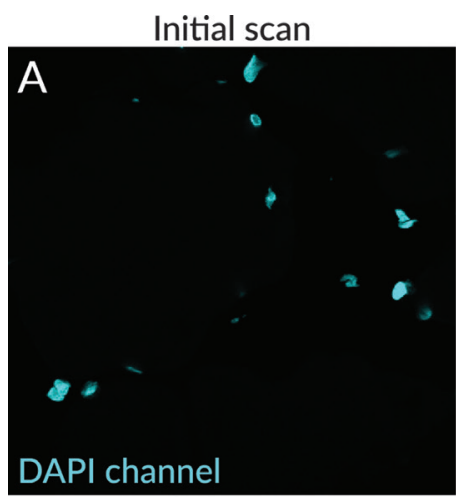

$405 \mathrm{~nm}$ laser, $10 \%, 60 \mathrm{sec}$.

$405 \mathrm{~nm}$ laser, 90\%, $60 \mathrm{sec}$.
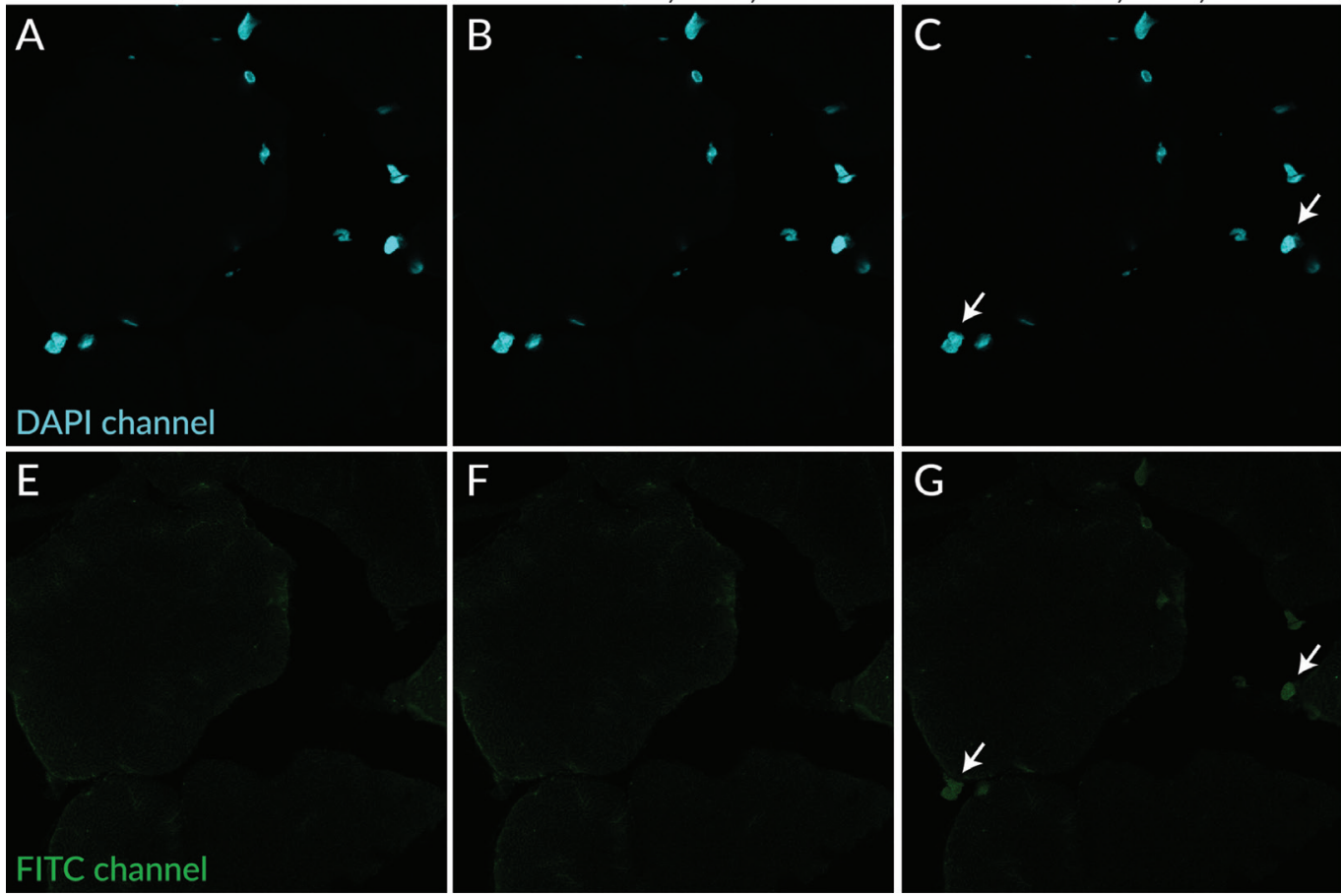

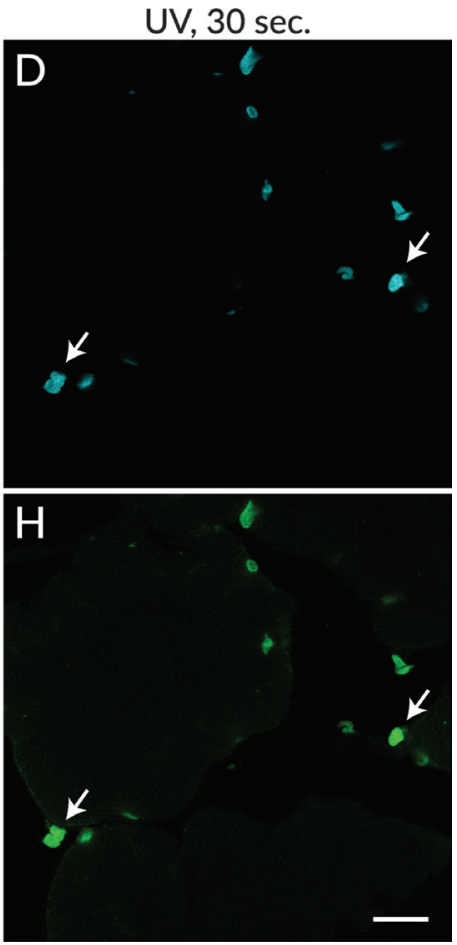

Figure 3: Photoconversion of DAPI was less apparent after exposure to $405 \mathrm{~nm}$ laser excitation compared to UV excitation. DAPI signal is shown in cyan (A-D). No green nuclear fluorescence was detectable in a field scanned without UV excitation (E), or after 60 seconds of exposure to $405 \mathrm{~nm}$ excitation at $10 \%$ power (F). Faint green nuclear signal was detected in the same field after subsequent exposure to 60 seconds of $405 \mathrm{~nm}$ excitation at $90 \%$ laser power (G, arrows). Green nuclear signal was readily detectable in the same field after subsequent exposure to UV light for 30 seconds $(D, H$, arrows indicate the same nuclei as in G). Scale bar $=20 \mu \mathrm{m}$. 
EverBrite $^{T M}$ Mounting Medium

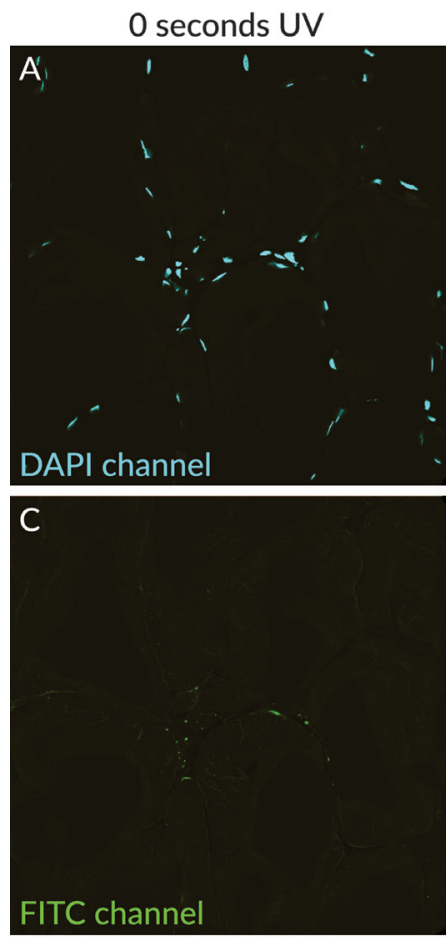

30 seconds UV
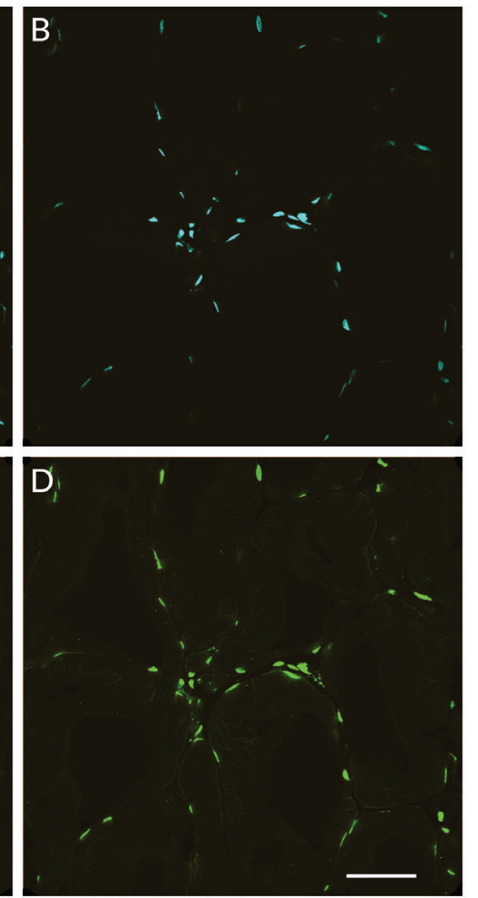

Drop-n-Stain EverBrite ${ }^{\mathrm{TM}}$ Mounting Medium

0 seconds UV

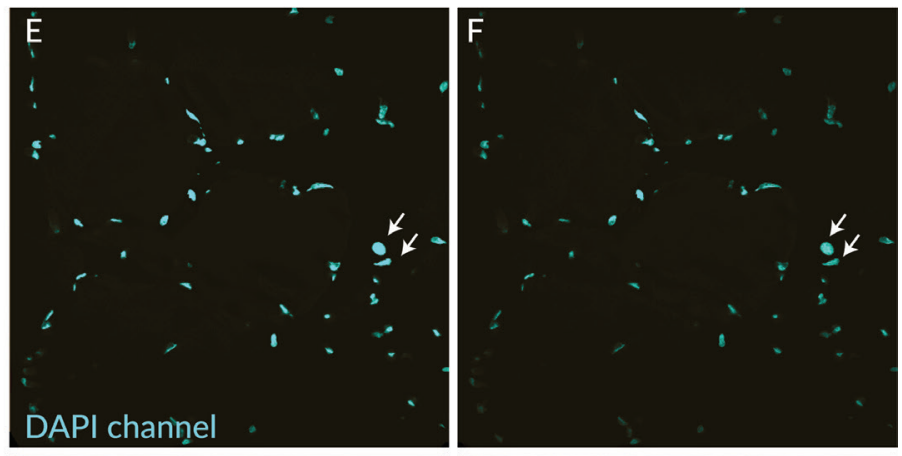

$\mathrm{H}$

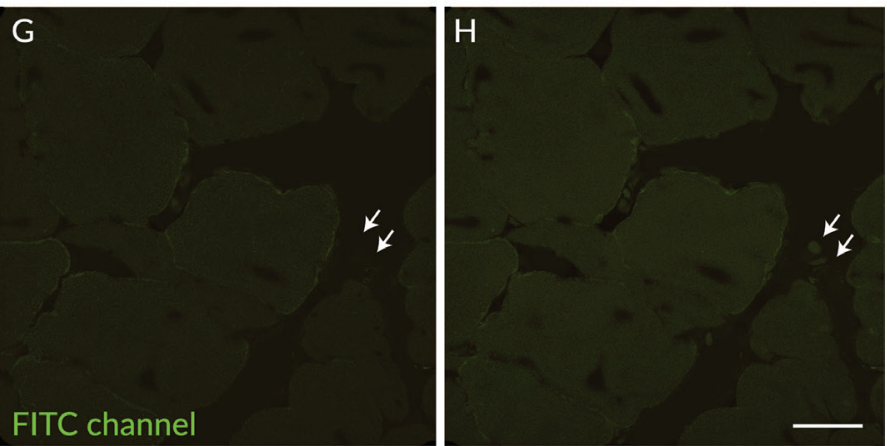

EverBrite $^{\mathrm{TM}}$ HardSet Mounting Medium

\section{0 seconds UV}

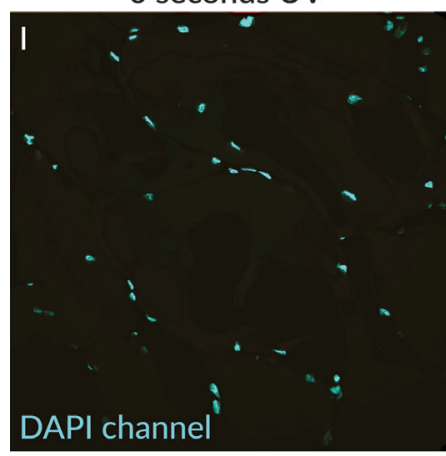

K

FITC channel
30 seconds UV

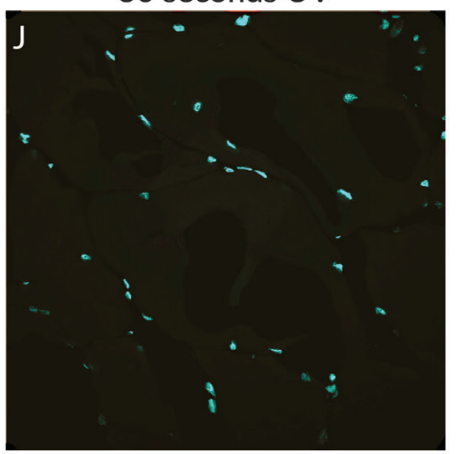

DAF

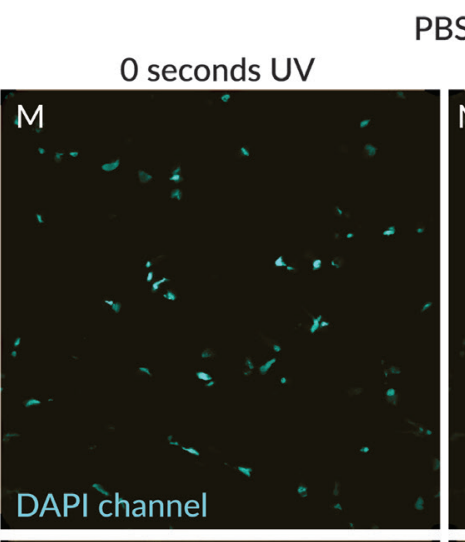

PBS

N

30 seconds UV

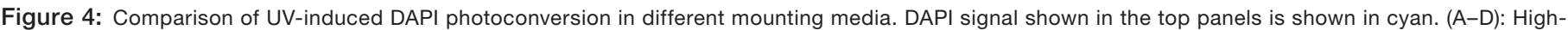

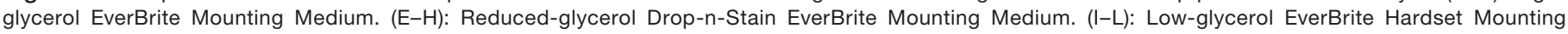

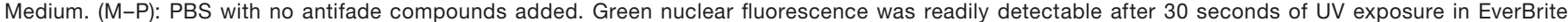

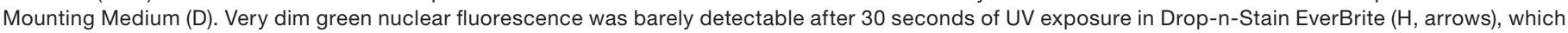

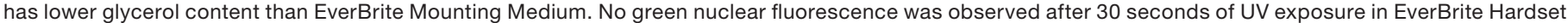
(L) or PBS (P). Scale bars $=50 \mu \mathrm{m}$. 
Specimen preparation effects. Ultra-violet exposure was performed on sections mounted with different antifade formulations or PBS. EverBrite Mounting Medium is a glycerolbased wet-set medium, whereas Drop-n-Stain EverBrite is a formulation with reduced glycerol content. EverBrite Hardset is a hardening low-glycerol formulation. After 30 seconds of UV exposure, conspicuous green fluorescent photoconversion product was observed in high-glycerol EverBrite medium (Figure 4D). In reduced-glycerol Drop-n-Stain EverBrite medium, very dim nuclear photoconversion fluorescence was barely detectable (Figure $4 \mathrm{H}$, arrows). Photoconversion of DAPI was not observed after 30 seconds of UV exposure in EverBrite Hardset or PBS (Figures 4L and 4P), although an increase in non-nuclear tissue autofluorescence was seen after UV exposure in EverBrite Hardset (Figure 4L). The fluorescence intensity of photoconversion product did not appear to correlate with the initial intensity of DAPI signal or the degree of DAPI photobleaching. For example, initial nuclear fluorescence intensity in the DAPI channel was comparable in sections mounted with EverBrite wet-set medium (Figure 4A) where photoconversion was readily detectable (Figure 4D) and with EverBrite Hardset medium (Figure 4I) where photoconversion was not observed (Figure 4L). Photobleaching of fluorescence in the DAPI channel was most pronounced in the section mounted in PBS (Figures $4 \mathrm{M}$ and $4 \mathrm{~N}$ ) where photoconversion was not detected (Figure 4P).

\section{Discussion}

Despite several published studies reporting the photoconversion of blue fluorescent nuclear dyes to form green and red fluorescent products, some microscopists may still be unaware of the potential for artifacts when using these near-ubiquitous stains. However, once the phenomenon is recognized, problems arising from photoconversion can be avoided through careful imaging practices, or by using different stains or mounting media.

Keep it short. The rate of photoconversion of DAPI and similar dyes may vary depending on instrument configuration. While photoconversion of DAPI can become apparent after a few seconds [2], detection of intense photoconversion product fluorescence more commonly requires continuous UV irradiation for 30 seconds or longer $[1,3,6]$. Photoconversion artifacts can be avoided by keeping UV excitation of DAPI as brief as possible when locating and focusing on specimens. Images of the nuclear stain should be captured last, after images are collected for other fluorophores. Although $405 \mathrm{~nm}$ laser excitation can induce photoconversion as previously reported [4], it required longer exposure time and high laser power (Figure 3). When using confocal microscopy, one can avoid photoconversion by navigating to fields not exposed to UV light before capturing images.

Change your reagents. Fixation method may affect photoconversion; however, published reports do not consistently agree on whether formaldehyde-fixed samples show less DAPI photoconversion than alcohol-fixed samples [1,2]. Glycerol, a common component of antifade mounting medium, has been reported to facilitate photoconversion [2], and in these experiments, photoconversion was readily observed only in highglycerol mounting medium (Figure 4). While the mechanisms of glycerol enhancement of photoconversion remain unclear, switching to a low-glycerol mounting medium may prevent the problem.

Published studies do not report consistent differences in photoconversion among DAPI, Hoechst, and other blue fluorescent stains. Using a far-red nuclear stain like RedDot ${ }^{\mathrm{m} x} 2$, NucSpot ${ }^{\circledR}$ Live 650, Draq5 $5^{\mathrm{m}}$, or SiR-DNA avoids the use of DAPI and Hoechst altogether. Like blue dyes, far-red dyes leave the green and visible red channels open for other fluorophores, but they are more costly than DAPI or Hoechst. Another disadvantage of far-red nuclear stains is that their fluorescence emission is not visible to the human eye, so they can't be used to locate and focus on specimens through the eyepieces.

Include controls for cross talk. While photoconversion can be a significant source of fluorescence cross talk with nuclear stains, it is by no means the major source of cross talk or bleed-through with these stains. DAPI and Hoechst dyes have broad fluorescence emission that overlaps substantially with green fluorescence emission. Because the dyes are not efficiently excited at $488 \mathrm{~nm}$, they can be well separated from green emitters by exciting the dyes separately. However, if blue and green fluorophores are excited simultaneously, DAPI will fluoresce brightly in the green emission channel.

Aside from nuclear stains, even spectrally well-separated fluorescent dyes used for multiplexing can show fluorescence cross talk, particularly if dyes are imaged simultaneously, or if staining with one fluorophore is much more intense than the other fluorophores. The recognition of photoconversion as another source of imaging artifacts highlights the importance of including single-stained controls to assess cross talk in multicolor fluorescence imaging experiments.

\section{Conclusion}

Photoconversion of DAPI, Hoechst, and similar dyes has been confirmed by several laboratories. Researchers should be aware of the potential for dye photoconversion in order to prevent it during imaging. However, photoconversion represents only one of multiple sources of fluorescence cross talk. Appropriate design of negative controls is essential to avoid cross talk artifacts, regardless of the dyes used.

\section{Acknowledgments}

The author thanks Alexis Madrid, James Maclean, and Daniel Wasik for critical reading of the manuscript.

\section{References}

[1] M Piterburg et al., J Microsc 246(1) (2012) 89-95.

[2] M Jež et al., Histochem Cell Biol 139 (2013) 195-204.

[3] TJ Karg and KG Golic, Chromosoma 127(2) (2018) $235-45$.

[4] V Hurst and SM Gasser, F1000Research 8 (2019) 104.

[5] D Żurek-Biesiada et al., Cytometry 83A(5) (2013) 441-51.

[6] S Rodic et al., McGill Sic Undergrad Res $J 9$ (2014) 31-40. 


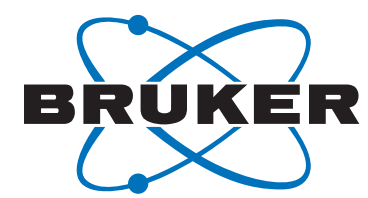

\section{Proven}

\section{Electron Microscopy} Solutions

\section{QUANTAX EDS}

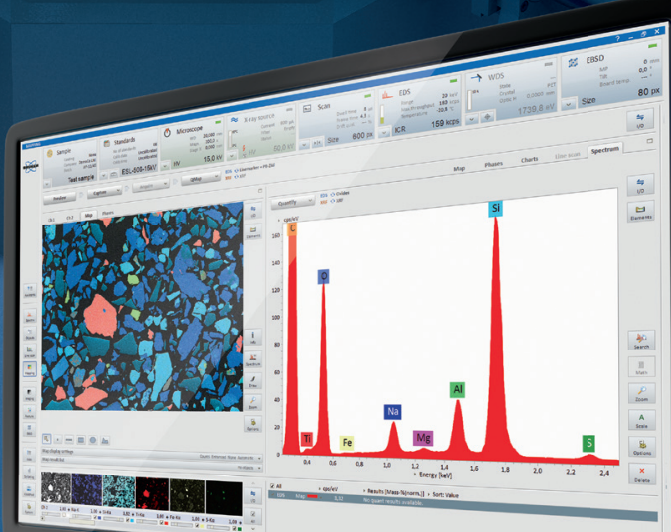

- Featuring XFlash ${ }^{\circledR} 6$ - worldwide leading SDD technology for SEM and TEM

- Delivering the fastest, most accurate EDS results

- Easiest-to-use EDS, available in configurations for every budget

- Integration of EDS, WDS, EBSD and Micro-XRF on SEM under a single user interface

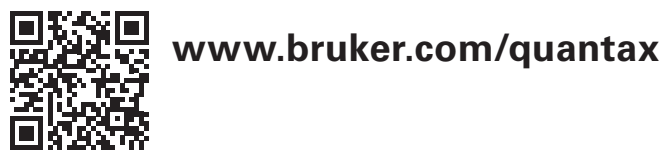

Innovation with Integrity 